\title{
CARDIAC GLYCOSIDE PLANTS SELF-POISONING
}

\author{
J. Radenkova-Saeva ${ }^{1}$ and P. Atanasov ${ }^{2}$ \\ ${ }^{1}$ Clinic of Toxicology, UMHATEM "N. I. Pirogov", Sofia, Bulgaria \\ ${ }^{2}$ Clinic of Internal Medicine, UMHATEM "N. I. Pirogov", Sofia, Bulgaria
}

\begin{abstract}
Summary. Cardiac glycosides are found in a diverse group of plants including Digitalis purpurea and Digitalis lanata (foxgloves), Nerium oleander, Convallaria majalis (lily of the valley), Strophanthus gratus, etc. Nerium Oleander is an indoor and ornamental plant of an evergreen shrub. It's widespread in countries with a Mediterranean climate. Oleander is one of the most poisonous plants known to humans. All parts of the nerium oleander are poisonous, primarily due to the contained cardiac glycosides - oleandrin, nerin, digitoxigenin, and olinerin of which oleandrin is the principal toxin. The bark contains the toxic substances of rosagenin which causes strychnine-like effects. Signs of poisoning appear a few hours after the adoption of the parts of the plant. Two cases of Nerium Oleander poisoning were presented. Clinical picture included gastrointestinal, cardiovascular and central nervous system effects. The clinical symptoms were characterized by nausea, vomiting, salivation, colic, diarrhoea, ventricular tachycardia, dysrhythmia, heart block, ataxia, drowsiness, muscular tremor. Treatment included administration of activated charcoal, symptomatic and supportive care.
\end{abstract}

Key words: toxic plants, oleander, poisoning, glycosides, oleandrin

\section{INTRODUCTION}

ardiac glycosides are found in a diverse group of plants including Digitalis purpurea and Digitalis lanata (foxgloves), Nerium oleander (common oleander), Thevetia peruviana (yellow oleander), Convallaria majalis (lily of the valley), Urginea maritima and Urginea indica (squill), Strophanthus gratus (ouabain), Apocynum cannabinum (dogbane), and Cheiranthus cheiri (wallflower).

Ancient Egyptians and Romans first used plants containing cardiac glycosides medicinally as emetics and for heart ailments. Toxicity from herbal cardiac glycosides was well recognized by 1785 , when William Withering published his classic 
work describing therapeutic uses and toxicity of foxglove, $D$ purpurea [1]. Toxicity may occur after consuming teas brewed from plant parts or after consuming leaves, flowers, or seeds from plants containing cardiac glycosides. Significant toxicity usually is a result of suicide attempt or inappropriate self-administration for the therapeutic purposes.

Nerium Oleander is an evergreen shrub reaching four metres in height. Leaves are 10 to $22 \mathrm{~cm}$ long narrow. The plant produces terminal flower heads, usually pink or white, however, 400 cultivars have been bred and these display a wide variety of different flower colour: deep to pale pink, lilac, carmine, purple, salmon, apricot, copper, orange. $\mathrm{N}$. oleander is cultivated worldwide as an ornamental plant; it is native only in the Mediterranean region $[4,5]$.

All parts of the nerium oleander are poisonous, primarily due to the contained cardiac glycosides - oleandrin, nerin, digitoxigenin, and olinerin of which oleandrin is the principal toxin. The bark contains the toxic substances of rosagenin which causes strychnine-like effects. Several flavones $(0.5 \%)$ and volatile oils (unimportant amount), as well as rubber, fats, sugars and hydrocyanic acid, can be isolated from its leaves $[4,5,7]$.

Cardiac glycosides are very similar to the toxin of foxglove. They have positive inotropic, negative chronotropic, and cross reactivity. This includes direct glycoside poisoning of the sodium-potassium pump of the heart and increased vagotonia. Most symptoms from oleander poisoning are cardiac and gastrointestinal in nature and appear four hours after the ingestion [7, 8].

Preparations containing the active principles of $\mathrm{N}$. oleander were used formerly as rodenticides, insecticides, and as remedies for indigestion, fever, malaria, leprosy, venereal diseases and as abortifacients.

Herein, we present two cases of Nerium oleander poisoning.

\section{CASE SERIES}

Case 1. A non-fatal case of Nerium oleander self-poisoning in a 73-year-old female is presented.

A patient drank two caps of herbal tea, prepared accidentally from Nerium oleander. Initial symptoms were nausea and vomiting, that appeared 30 minutes later. Central nervous system effects occurred later and included ataxia, drowsiness, muscular tremor.

The patient arrived to the hospital around 3 hours after the accident. She complained of abdominal pain, palpitation, breathing difficulty.

Previous history included arterial hypertension, coxarthrosis, depression disorder.

In the Emergency Department Toxicology Unit vital signs were as follows: temperature $-36.5^{\circ} \mathrm{C}$, pulse 141 beats/minute; respiratory rate 28 breaths/minute; blood pressure $177 / 115 ; 138 / 83 \mathrm{~mm} \mathrm{Hg} ; \mathrm{O}_{2}$ Sat $96.1 \%$.

On physical examination the patient was drowsy, with pallor skin. The pupils were normally wide. Lungs had clear breath sounds. Heart sounds - with no mur- 
mur, or rub. Abdomen was soft, no guarding or rebound. No hepatosplenomegaly was noted. Extremities -without edema.

No abnormalities were detected on chest $X$ - ray studies.

ECG demonstrated transitional right bundle branch block (RBBB), on the background of racing heart activity and single ventricular extrasystoles.

During the course of the treatment, with normalization of heart frequency, cardiovascular disturbances progressed to atrioventricular (AV) block - I degree, with periods of disturber conductivity on right bundle to complete restore of ECG image. In the period of restoration of the ECG image, we observed transitional abnormality in repolarization as ST depression of up to $3 \mathrm{~mm}$, without other electrophysiological abnormalities.

Laboratory data: Hematology studies: $\mathrm{Hb}-141$, Hct 0.40, Leuc 13.7. Chemistry: urea, creatinine, electrolytes, total protein, albumin tests were unremarkable, total bilirubin - $23.5 \mathrm{mcmol} / \mathrm{l}$, blood glucose $-7.2 \mathrm{mmol} / \mathrm{l}$. Enzyme tests: ALT, AST - unremarkable, CPK - 249 U/I, CPK-MB - 8,4 U/l; INR - 1.06.

The management protocol followed the one adopted by the Intensive Care Department and consisted of monitoring of various parameters such as: continuous ECG monitoring, pulse rate, arterial pressure, body temperature, percentage of oxygen-haemoglobin saturation, urine output, etc.

Intensive care was given. Treatment consisted of activated charcoal administration, fluid infusion $1000 \mathrm{ml}$, insulin 12 units, Betaloc Zok tabl. 50, $25 \mathrm{mg}$, Urbason 40; 20 mg i.v., Fragmin - 2500E, Vit. C 500 mg iv.

The patient remained in the hospital for three days. ECG normalized. Betaloc Zok tabl. $25 \mathrm{mg}$ was continued at home.

Case 2. A 59-year-old male was admitted in the emergency toxicology room with vomiting and lightheadedness, 2 hours after ingestion of common oleander aqueous leaf extract. He took the extract with suicidal intentions.

On initial examination, the blood pressure was $95 / 60 \mathrm{~mm} \mathrm{Hg}$ with regular pulse of $60 \mathrm{bpm}$. He was looking toxic due to excessive vomiting. Other general physical parameters were normal. His chest and lungs were clear on auscultation and percussion.

Electrocardiogram revealed sinus rhythm.

Laboratory data: Hematology studies: $\mathrm{Hb}-147$, Hct -0.44 ; Leuc - 11.2. Chemistry: urea, creatinine, electrolytes, total protein, blood glucose, albumin tests were within the reference range, total bilirubin $-37 \mathrm{mcmol} / \mathrm{l}$. Enzyme tests: ALT, AST - in reference range, $\mathrm{CPK}-278 \mathrm{U} / \mathrm{l}$.

Treatment consisted of activated charcoal, fluid infusion, atropine, Urbason, Quamatel.

\section{DISCUSSION}

The poisonous effect of the oleander has been well known for centuries. There are data relating to its having poisoned Napoleon soldiers during one of their campaigns. 
The clinical characteristics of intoxication are mainly cardiac and gastrointestinal.

The cardiac effects of the glycosides are due to direct cardiotoxicity and an indirect effect via the vagal nerve. The direct effect is due to the inhibition of the Na-K ATP-ase pump (sodium-potassium adenosine triphosphatase enzyme system). This specific action increases intracellular sodium ion and serum potassium concentrations. The sodium influx lowers the membrane potential threshold, increasing excitability.

In myocytes, elevated intracellular sodium concentrations produce increased intracellular calcium concentrations via a $\mathrm{Na}^{+}$-calcium $\left(\mathrm{Ca}^{++}\right)$-exchanger. In response to the increased intracellular calcium, the sarcoplasmic reticulum releases additional calcium intracellularly resulting in depolarization of the cell.

As a result of this excessive intracellular calcium, enhanced cardiac contractions, which are delayed after depolarizations, occur. These clinically manifest as aftercontractions, such as premature ventricular contractions (PVCs). Cardiac glycosides also have vagotonic effects, resulting in bradycardia and heart blocks. Inhibition of $\mathrm{Na}^{+}-\mathrm{K}^{+}$-ATPase in skeletal muscle results in increased extracellular potassium and contributes to hyperkalemia.

Cardiac glycosides primarily affect cardiovascular, neurologic, and gastrointestinal systems. Of these, effects on the cardiac system are most significant. The pathophysiology that produces cardiotoxicity involves prolonging refractory period in atrioventricular (AV) node, shortening refractory periods in atria and ventricles, and decreasing resting membrane potential (increased excitability). At therapeutic doses, cardiac glycosides also may increase inotropy. Any dysrhythmia characterized by both increased automaticity and depressed conduction is suggestive of cardiac glycoside toxicity.

Sinus rhythm with PVCs is the most common rhythm associated with digitalis toxicity. Other dysrhythmias often associated with cardiac glycoside toxicity include bradydysrhythmias, sinus bradycardia with all types of AV nodal block, junctional rhythms, and sinus arrest. Dysrhythmias characterized by increased automaticity and conduction blockade, when combined, are highly suggestive of cardiac toxicity. These dysrhythmias include the following: tachydysrhythmias, such as atrial tachycardia with block; junctional tachycardia; ventricular tachycardia; ventricular fibrillation; paroxysmal atrial tachycardia with block; bidirectional ventricular tachycardia.

The clinical effects are mainly cardiac and gastrointestinal, resulting in a clinical picture similar to that of digoxin poisoning, including nausea, vomiting, salivation, colic and diarrhoea $[6,10]$.

Sinus bradycardia is the most frequent cardiovascular sign, and it may progress to atrioventricular block (AV) and asystole. Ventricular arrhythmias, premature ventricular beats, ventricular tachycardia and ventricular fibrillation can occur in severe cases [9].

Continuous ECG monitoring for at least $24 \mathrm{~h}$ is necessary to detect arrhythmias; longer monitoring is appropriate in patients with severe poisoning [9]. Pa- 
tients with cardiovascular illness, and/or those who arrive late to hospital are at highest risk. Serum potassium concentrations are of prognostic importance, as in digoxin intoxication. The presence of hyperkalaemia in the initial stage of intoxication worsens the prognosis. Deaths from ingestion of $\mathrm{N}$. oleander occur due to dysrhythmias [6, 8].

The treatment of oleander poisoning is empirically based on the treatment of digitalis-glycoside toxicity and consists of supporting the patient hemodynamically. This may include administering atropine for severe bradycardia; using phenytoin or lidocaine hydrochloride to control dysrhythmias; placing a temporary venous pacemaker; or electrical counter shock.

Supportive care. Correction of dehydration with normal saline is necessary, and antiemetics are used to control severe vomiting.

Electrolytes. Hypokalemia worsens toxicity due to digitalis glycosides, and hyperkalemia is life-threatening. Both must be corrected. Hyperkalemia is due to extracellular shift of potassium rather than an increase in total body potassium and is best treated with insulin-dextrose infusion.

Gastric decontamination. The place of emesis induction and gastric lavage has not been investigated, although they are used in practice. Single-dose activated charcoal is probably beneficial.

Digoxin-specific antibody fragments. Digoxin-specific antibody fragments are effective in reverting life-threatening cardiac arrhythmias; prospective observational studies show a beneficial effect on mortality. High cost and lack of availability limit the widespread use of digoxin-specific antibody fragments in developing countries [2, 3].

\section{CONCLUSION}

Two non-fatal cases of oral administration of Nerium Oleander extract have been described. Early diagnosis, decontamination and subsequent treatment may have played an important role for the successful management.

\section{REFERENCES}

1. Bessen, H. A. Therapeutic and toxic effects of digitalis: William Withering, 1785. - J. Emerg. Med., 4, 1986, № 3, 243.

2. B o u rg e o is, B., P. Incagnoli, J. Hanna etV. Tirard. Traitement par anticorps antidigitalique d'une intoxication volontaire par laurier rose. - Annales Françaises d'Anesthésie et de Réanimation, 24, 2005, № 6, 640-642.

3. Camphausen, C., N. A. Haas et A. C. Mattke. Successful treatment of oleander intoxication (cardiac glycosides) with digoxin-specific Fab antibody fragments in a 7-year-old child. - Zeitschrift für Kardiologie, 94, 2005, № 12, 817-823.

4. Grae me, K. Toxic plant ingestions. In: Auerbach PS, ed. Wilderness Medicine, Chapter 58, 5th Ed. Philadelphia, Pa: Mosby Elsevier, 2007. 
5. Hostetle r, M. et S. Schreiber. Poisonous plants. In: Tintinalli JE, Kelen GD, Stapczynski JS, Ma OJ, Cline DM, eds. Emergency Medicine: A Comprehensive Study Guide. Chapter 205, 6th ed. New York, NY: McGraw-Hill, 2004.

6. Le Couteur, D. G. et A. A. Fisher. Chronic and criminal administration of Nerium oleander. - J. Toxicol. Clin. Toxicol., 40, 2002, № 4, 523-524.

7. Sh a rm a, P. et al. Chemical Constituents of Plants from the Genus Nerium. - Chemistry \& Biodiversity, 7, 2010, № 5, 1198-1207.

8. Lang ford S. D. et P. J. Boor. Oleander toxicity: an examination of human and animal toxic exposures. - Toxicology, 109, 1996, № 1, 1-13.

9. Senthilkumaran, S. et al. (2011). Electrocardiographic changes during inhalational oleander toxicity. - J. Electrocardiol., 44, 2011, № 4, 470-472.

10. Wa s fi, l. et al. (2008). A fatal case of oleandrin poisoning. - Forensic Science Int., 179, 2008, № 2-3, e31-36.

- Corresponding author:

Assoc. Prof. Julia Radenkova-Saeva, MD, PhD

Clinic of Toxicology

UMHATEM „N. I. Pirogov”

21 Totleben Blvd.

$\mathrm{Bg}-1606$ Sofia

e-mail: jrsaeva2@yahoo.co.uk 